\title{
Constructive role of noise: Fast fluctuation asymptotics of transport in stochastic ratchets
}

\author{
Charles R. Doering and Lubomira A. Dontcheva \\ Department of Mathematics, University of Michigan, Ann Arbor, Michigan 48109-1109 \\ Malgorzata M. Klosek \\ Department of Mathematical Sciences, University of Wisconsin, Milwaukee, Wisconsin 53201
}

(Received 20 January 1998; accepted for publication 1 May 1998)

\begin{abstract}
The constructive role of random fluctuations is studied in the context of transport in stochastic ratchets. We discuss the interplay of independent white (thermal) and discrete (external) noises and their generation of transport in anisotropic potentials. The constructive cooperation of such fluctuations is most apparent in the asymptotic limit of fast discrete-valued noise, a limit which presents some interesting mathematical features. We describe the asymptotic analysis of the current in the limit of fast external noise, pointing out the strong qualitative dependence of the current on the interplay of the independent noise sources and its surprising sensitivity to the regularity of the underlying anisotropic ratchet potential. (C) 1998 American Institute of Physics.
\end{abstract}

[S1054-1500(98)01103-3]

The interplay of the effects of independent sources of noise in a simple nonlinear dynamical system is investigated via asymptotic analysis of a model "stochastic ratchet." For discrete noise-perturbed diffusion in an anisotropic periodic potential, the resulting nonequilibrium current is found to depend strongly on the degree of regularity (smoothness) of the potential. In the presence of order one diffusion, continuous ratchet forces along with an additive discrete-valued noise force lead to, in the asymptotically fast discrete noise limit, a certain scaling of the current. We find, perhaps somewhat surprisingly, that discontinuous ratchet forces produce a different scaling. This analysis sheds new light on some mathematical aspects of models that have attracted attention in condensed matter and biological physics in recent years.

\section{INTRODUCTION}

It has long been recognized that noise and fluctuations can play a crucial role in transport processes, the canonical example being thermal activation. In the usual Kamers picture $^{1}$ of noise-activated rate processes, transport between metastable states proceeds either towards a thermal equilibrium distribution for "isolated" systems or to a nonequilibrium steady state for "externally" driven systems. By "isolated" systems we mean those whose stochastic evolution is derived from an underlying time-reversal invariant dynamics. Their stochastic dynamics then obey detailed balance, so the associated steady states are true time-reversal invariant equilibrium states so no net transport is possible beyond transient displacements. Broad classes of subsystems coupled to an equilibrium thermal bath provide examples of such systems, where the thermal fluctuations are modelled by stochastic noise processes.

On the other hand, systems appropriately perturbed by "external" forces may possess statistical steady states with a flux, or flow. An obvious way to realize such a state is to apply a uniform force $F$ to a system of particles, which in many cases results in a net current of particles or probability proportional to $\mu F$, where $\mu$ is an appropriate mobility which may itself depend on $F$ and the system's internal structure and fluctuations. ${ }^{2}$ In the past few years there has been a tremendous amount of interest in so-called "ratchet", models ${ }^{3}$ where spatially uniform mean-zero symmetrically distributed time-dependent forces, which may be random or periodic in time, interact with an underlying spatial anisotropy to generate motion. Such fluctuating force ratchet models display some subtle features such as unexpected current reversals as a function of numerous parameters ${ }^{4}$ and a remarkably sensitive dependence on the nature of the fluctuating forces and the regularity of the anisotropic potential. ${ }^{5}$ It is the purpose of this paper to describe and discuss the latter phenomena, in particular the mathematical issues related to the fast-noise asymptotic calculation of the current in the simplest fluctuating force ratchet model. These investigations uncover a strong cooperative effect between white (internal, thermal) noise and independent fast discrete (external) noise in stimulating transport in stochastic ratchets.

These and related fluctuating potential ${ }^{6}$ ratchet systems have been used to model fundamental mechanisms for subcellular biological transport processes, ${ }^{7}$ and investigated experimentally as mesoscopic particle manipulation techniques. ${ }^{8}$ Several more general review papers have appeared in recent years, ${ }^{9}$ and we refer the interested reader to those for a more complete description of the models, applications, experiments, and reversal phenomena. In this paper we will concentrate on what may be the simplest and ostensibly most straightforward ratchet model, that of a particle in one dimension subject to a dichotomous Markov (a.k.a. external) force and/or independent white (a.k.a. internal, or thermal) noise. We describe the asymptotic analysis of the 
current in the limit of fast external noise, pointing out a very strong qualitative dependence of the current on the interplay of the independent noise sources and the details of the underlying anisotropic ratchet potential. Denoting the small time scale of the external noise by $\tau$, we may summarize our results as follows: as $\tau \rightarrow 0$, (a) the current is exponentially small, $O\left(e^{-C / \tau}\right)$ with $C>0$, in the absence of thermal noise while it is only algebraically small, $O\left(\tau^{\beta}\right)$ with $\beta>0$, in the presence of white noise, and (b) in the cases where it is algebraically small, the scaling exponent $\beta$ may depend on the regularity of the ratchet potential $\beta=3$ for continuous forces, $\beta=\frac{5}{2}$ for discontinuous forces). These results illustrate some interesting and rather delicate aspects of the mathematical issues surrounding the analysis of nonequilibrium stochastic dynamic models in general, and ratchet models in particular.

The remainder of this paper is organized as follows. In the next section we describe the mathematical model that is the focus of our study and review some elementary features of its behavior. In Sec. III we describe the asymptotic $\tau$ $\rightarrow 0$ limit of the nonequilibrium current for various cases. Although there is also a natural white-noise limit for the discrete process wherein the amplitude of the noise is appropriately increased as $\tau \rightarrow 0$ [see, for example, the analyses in Refs. 4(a) and 5], we will concentrate on the fast external noise limit where the amplitude remains $O(1)$. In Sec. IV we summarize our results and discuss some implications for other systems.

\section{SIMPLE MODELS AND MECHANISMS}

Consider an overdamped particle moving on a ring, $x$ $\in[0, L]$ with 0 and $L$ identified, under the influence of a force $f(x)$ derived from a periodic potential $V(x)$ $=-\int_{0}^{x} f(y) d y$ and white noise of intensity $D$ :

$$
\begin{aligned}
& \frac{d x}{d t}=f(x)+\sqrt{2 D} \xi(t), \quad\left\langle\xi(t) \xi\left(t^{\prime}\right)\right\rangle=\delta\left(t-t^{\prime}\right), \\
& x(0)=x_{0} .
\end{aligned}
$$

The time-dependent probability density, $\rho(x, t)$, obeys the Fokker-Planck-Smoluchowski equation

$$
\frac{\partial \rho}{\partial t}=\partial_{x}\left\{V^{\prime}+D \partial_{x}\right\} \rho
$$

with periodic boundary conditions in $x$ and initial probability distribution $\rho(x, 0)=\delta\left(x-x_{0}\right)$. The evolution for the probability distribution may be rewritten as a continuity equation,

$$
\frac{\partial \rho}{\partial t}+\partial_{x} J=0,
$$

where we identify the local instantaneous probability current

$$
J(x, t)=f(x) \rho(x, t)-D \partial_{x} \rho(x, t) .
$$

The instantaneous noise-ensemble averaged particle velocity is the integral of $J$ :

$$
\left\langle\frac{d x}{d t}\right\rangle=\int_{0}^{L} J(x, t) d x .
$$

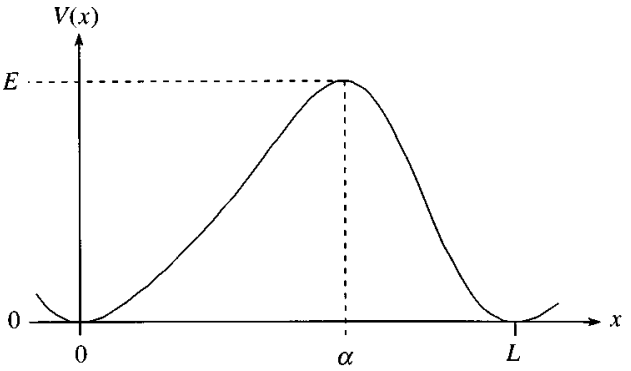

(a)

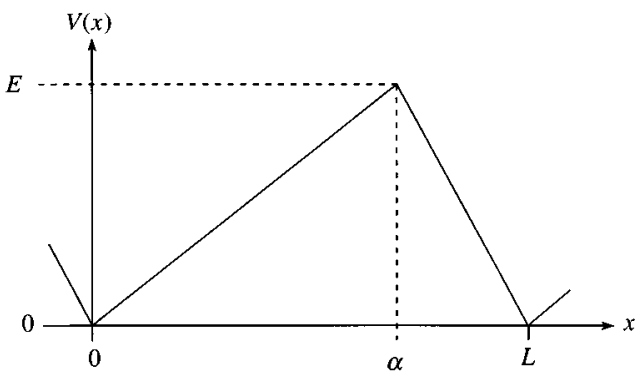

(b)

FIG. 1. Ratchet potenials $V(x)$. In (a) we illustrate a smooth potential, and in (b) a piecewise linear potential with discontinuous force. The energy barrier height is $E$ and the parameter $\alpha \neq L / 2$ indicates the anisotropy.

What we have in mind for the potential $V(x)$ is a periodic anisotropic potential like the one shown in Fig. 1, which may be smooth or piecewise smooth with a discontinuous force. Under very general conditions the probability distribution relaxes, as $t \rightarrow \infty$, to the stationary probability distribution

$$
\rho_{\mathrm{eq}}(x)=\frac{1}{Z} e^{-V(x) / D}, \quad Z=\int_{0}^{L} e^{-V\left(x^{\prime}\right) / D} d x^{\prime} .
$$

This equilibrium Gibbs distribution leads to the natural identification of the white noise as thermal fluctuations, and to the particle diffusion coefficient $D$ as $k_{\mathrm{B}} T$, Boltzmann's constant times the temperature.

Despite any anisotropy in $V(x)$, this distribution supports no current,

$$
J_{\text {eq }}=f(x) \rho_{\text {eq }}(x)-D \partial_{x} \rho_{\text {eq }}(x)=0,
$$

so the average particle velocity (net drift) vanishes in the steady state:

$$
\left\langle\frac{d x}{d t}\right\rangle \rightarrow 0 \text { as } t \rightarrow \infty \text {. }
$$

The simplest example of a nonequilibrium steady state arises if an additional uniform force $F$ is also applied to the particle so the equation of motion is

$$
\frac{d x}{d t}=f(x)+F+\sqrt{2 D} \xi(t) .
$$

The probability density must still be periodic on $[0, L]$, but the "tilted" potential energy $V(x)-F x$ is not. Hence the steady-state current, $J_{\mathrm{ss}}$, which must be constant $\left(\partial_{x} J_{\mathrm{ss}}\right.$ 
$=0$ ), may not vanish. The straightforward solution of the differential equation for the steady-state probability density $\rho_{\mathrm{ss}}$, as in Ref. 2, yields the current

$$
J_{\mathrm{ss}}=\frac{D\left(1-e^{-F L / D}\right)}{\int_{0}^{L} e^{-(V(x)-F x) / D}\left(\int_{x}^{L} e^{(V(y)-F y) / D} d y\right) d x} .
$$

For large values of $|F|$, i.e., $|F| \gg \sup _{x}|f(x)|, J_{\mathrm{ss}} \sim F / L$. For small $|F|, J_{\mathrm{ss}} \sim \mu F$ with mobility

$$
\mu \sim \frac{L}{\int_{0}^{L} e^{-V(x) / D}\left(\int_{x}^{L} e^{V(y) / D} d y\right) d x} .
$$

In either case the transport mechanisms are clear: the particle effectively moves down an inclined washboard potential, limited by the friction for large $F$ and by the thermal activation rate over remaining barrier(s) for small $F$. Note that for all but the largest $|F|$, the magnitude of $J_{\mathrm{ss}}$ is generally not the same for $F$ and $-F$. If $V(x)$ were isotropic, i.e., if $V(x)=V(L-x)$, then $J_{\mathrm{ss}}(F)=-J_{\mathrm{ss}}(-F)$, but for anisotropic potentials this is not the case. It is this asymmetry that underlies the ratchet transport mechanism considered here.

The idea behind transport in a stochastic ratchet is one step removed from the simple diffusion in a tilted periodic potential. For a ratchet we consider an applied external force which is spatially homogeneous but time dependent and mean zero. Such a force could be a periodic or sinusoidal ac force, as in Ref. 4(b), but we will concentrate on two-level Markov processes so that the steady states for the overall system evolution can be studied in the framework of stationary Markov processes. In particular we consider the equation of motion

$$
\frac{d x}{d t}=f(x)+I(t)+\sqrt{2 D} \xi(t),
$$

where $I(t)$ takes on two values, $\pm F$, switching between the two levels at random, independent exponentially distributed times. The probabilities $p_{ \pm}(t)$ that $I(t)$ is in the \pm state at time $t$ evolve according to the master equation

$$
\frac{d}{d t}\left(\begin{array}{l}
p_{+} \\
p_{-}
\end{array}\right)=\frac{1}{2 \tau}\left(\begin{array}{cc}
-1 & 1 \\
1 & -1
\end{array}\right)\left(\begin{array}{l}
p_{+} \\
p_{-}
\end{array}\right) .
$$

The stationary correlation function of the external noise is

$$
\left\langle I(t) I\left(t^{\prime}\right)\right\rangle=F^{2} e^{-\left|t-t^{\prime}\right| / \tau},
$$

so we can identify the noise correlation time $\tau$ as the relevant noise time scale.

The enlarged stochastic process consisting of the position of the particle together with the state of the external force is a Markov process characterized by the probabilities $\rho_{ \pm}(x, t)$, the densities for the position variable given the \pm state of the force. These joint probabilities evolve according to the coupled Fokker-Planck-Smolukowski equations

$$
\begin{aligned}
\frac{\partial}{\partial t}\left(\begin{array}{l}
\rho_{+}(x, t) \\
\rho_{-}(x, t)
\end{array}\right) & \\
= & \left(\begin{array}{cc}
\partial_{x}\left\{V^{\prime}-F+D \partial_{x}\right\}-\frac{1}{2 \tau} & \frac{1}{2 \tau} \\
\frac{1}{2 \tau} & \partial_{x}\left\{V^{\prime}+F+D \partial_{x}\right\}-\frac{1}{2 \tau}
\end{array}\right) \\
& \times\left(\begin{array}{l}
\rho_{+}(x, t) \\
\rho_{-}(x, t)
\end{array}\right) .
\end{aligned}
$$

The average particle transport is determined by the current associated with the continuity equation for the marginal density of the position, $\rho(x, t)=\rho_{+}(x, t)+\rho_{-}(x, t)$. Adding together the evolution equations for $\rho_{ \pm}$we find

$$
\frac{\partial \rho}{\partial t}+\partial_{x} J=0
$$

with the current

$$
J=f(x) \rho-D \partial_{x} \rho+F\left(\rho_{+}-\rho_{-}\right) .
$$

The goal of the analysis, then, is to compute the steady-state probabilities $\rho_{ \pm}(x)$ by solving the time homogeneous equations

$$
\begin{aligned}
\left(\begin{array}{l}
0 \\
0
\end{array}\right) & =\left(\begin{array}{cc}
\partial_{x}\left\{V^{\prime}-F+D \partial_{x}\right\}-\frac{1}{2 \tau} & \frac{1}{2 \tau} \\
\frac{1}{2 \tau} & \partial_{x}\left\{V^{\prime}+F+D \partial_{x}\right\}-\frac{1}{2 \tau}
\end{array}\right) \\
& \times\left(\begin{array}{l}
\rho_{+}(x) \\
\rho_{-}(x)
\end{array}\right)
\end{aligned}
$$

with periodic boundary conditions for $x \in[0, L]$. The average particle drift velocity, i.e., the nonequilibrium transport, is then determined by the steady-state current according to Eq. (17), i.e., $\langle d x / d t\rangle=J L$. We are interested in the dependence of $J$ on the system parameters $D, \tau, F$, and functionally on $f(x)$.

The physical mechanism for particle transport under the influence of the mean zero fluctuating force is evident from the considerations mentioned around Eq. (8). For anisotropic periodic potentials the net drift in the \pm directions need not be, and in general is not, the same. Time reversal symmetry is broken by the spatial anisotropy together with the loss of detailed balance induced by the symmetric external force. For the relatively simple ratchet potentials in Fig. 1, the direction of the current may also be understood: the energy barrier for diffusion to the right when the force pushes to the right is lower than the energy barrier for diffusion to the left when the force pushes to the left, so in these cases we expect (and we will find) positive net drift to the right. (For more complicated shapes this intuition breaks down and we must resort to analysis of the model to determine not just the magnitude, but even the direction of the current.) And because the external force in this model does not react to the particle, switching regardless of the particle's position, it may expend 
net energy on the particle and hence perform useful work. Indeed, we could include a homogeneous "load force" on the system (a uniform bias) and, in cases where the induced drift opposes the load force, measure the net useful work exerted in the transport process.

Equation (16) is not exactly solvable in general, but we can deal with particular limiting cases. Of course in the limit $F=0$ there is no external fluctuating force, $\rho_{+} \rightarrow \rho_{-} \rightarrow \rho_{\mathrm{eq}}$, and the current vanishes. The power series expansion of $J$ for small $F$ may be obtained via a regular perturbation analysis of the differential equations.

In the limit $\tau \rightarrow \infty$, for example, the external force fluctuates so slowly that the equations for $\rho_{+}$and $\rho_{-}$decouple, becoming independent versions of Eq. (8). The net current is then the difference between the steady-state currents for $+F$ and $-F$. A systematic perturbation analysis for $J$ in powers of $\tau^{-n}$ for this case-also a regular perturbation expansion-is described in Ref. 9(a).

In the case that there is no thermal white noise, i.e., $D$ $=0$, the problem reduces to two coupled first-order ordinary differential equations which may be solved exactly, as was originally done in Ref. 4(a). For the situation $F$ $>\sup _{x}|f(x)|$, the current is

$$
J=\frac{1-e^{\phi(L)}}{Q},
$$

where

$$
\phi(x)=-\frac{1}{\tau} \int_{0}^{x} \frac{f(y)}{F^{2}-f(y)^{2}} d y
$$

and

$$
\begin{aligned}
Q= & \int_{0}^{L} \frac{e^{-\phi(x)}}{F^{2}-f(x)^{2}}\left(\int_{x}^{L} d y\left(\frac{1}{\tau}-V^{\prime \prime}(y)\right) e^{\phi(y)}\right) d x \\
& +e^{\phi(L)} \int_{0}^{L} \frac{e^{-\phi(x)}}{F^{2}-f(x)^{2}} \\
& \times\left(\int_{0}^{x} d y\left(\frac{1}{\tau}-V^{\prime \prime}(y)\right) e^{\phi(y)}\right) d x .
\end{aligned}
$$

This solution, although exact, is far from transparent: even the sign of $J$ is not obvious. For ratchet potentials as in Fig. 1 it does, in fact, yield a positive current. This is hinted at by the shape of the "effective" potential $\phi(x)$ as shown in Fig. 2 ; the ratchet potential $V(x)$ is periodic but $\phi(x)$ is not and its bias indicates the current-generating effect of the nonequilibrium fluctuations for these simple cases.

Other limits, particularly the $\tau \rightarrow 0$ fast noise limit, are more delicate. In the next section we focus on the analysis of this limit with particular interest paid to differences between the $D=0$ and the $D \neq 0$ situations and continuous and discontinuous force cases.

\section{FAST NOISE ASYMPTOTICS}

In this section we consider asymptotic expansions for the ratchet current $J$ in the fast noise limit, $\tau \rightarrow 0$ with all other parameters fixed. This is a singular limit of the differential equations for $\rho_{ \pm}$, and we will find some correspondingly

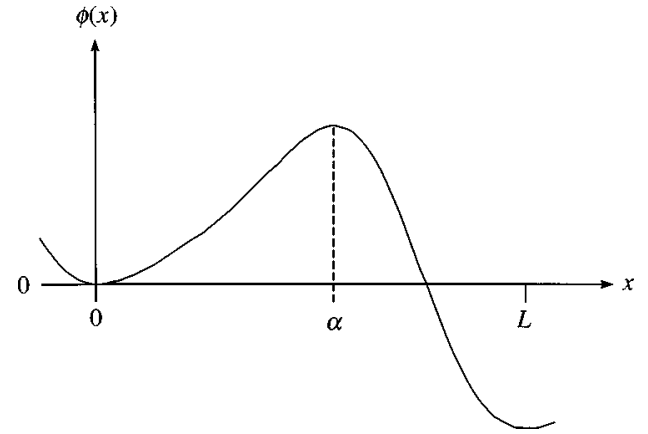

FIG. 2. A sketch of the "effective", potential $\phi(x)$, from Eq. (20), for ratchet potentials of the form of those in Fig. 1.

singular dependence of the current on the system variables. There are two cases of this limit which must be analyzed separately: for $D=0$ we have the exact solution which may be studied directly, while for $D \neq 0$ we must use singular perturbation methods on Eqs. (18). The extreme sensitivity of $J$ on $D$ will be the hallmark of the cooperative effect of the internal (white) and external noises. Additionally we will see that each of these cases has two subcases: for smooth ratchet potentials, i.e., with continuous $V^{\prime \prime}(x)=-f^{\prime}(x)$, there is one form of behavior, while for simple piecewise linear $V(x)$, i.e., discontinuous forces, there is another. In all cases we will only consider the relatively strong external force situation where $F>\sup _{x}|f(x)|$.

First consider the case $D=0$ and smooth ratchet potentials. The exact solution is given in Eqs. (19)-(21) and the fast noise limiting behavior of $J$ is obtained by direct asymptotic analysis of the integrals in the formula via Laplace's method. ${ }^{10}$ For the particular structure of the ratchet potential as in Fig. 1(b), with the corresponding structure for the "effective", potential $\phi(x)$ as in Fig. 2, the factor $e^{\phi(L)}$ is exponentially small and $e^{\phi(\alpha)}$ is exponentially large. All the integrals are dominated by contributions near $\alpha$ and/or $L$, and a straightforward accounting of all the terms yields the asymptotic formula

$$
J \sim \frac{1}{\pi} \sqrt{\left|V^{\prime \prime}(\alpha)\right|\left|V^{\prime \prime}(L)\right|} e^{-\phi(\alpha)},
$$

where for reference we recall that

$$
-\phi(\alpha)=\frac{1}{\tau} \int_{0}^{\alpha} \frac{f(x)}{F^{2}-f(x)^{2}} d x .
$$

Hence we observe an exponentially small current, $J$ $=O\left(e^{-C / \tau}\right)$ with a positive constant $C$ that depends on $F$ and functionally on $V(x)$. It is interesting that this expression, in particular the exponential term, only depends on the ratchet potential between $x=0$ and $x=\alpha$. That is, the current in this formula is independent of the details of the structure of $V(x)$ and $f(x)$ for $x$ between $\alpha$ and $L$. This suggests that as $t \rightarrow 0$ the transport process proceeds only to the right with essentially no "backslips" to the left. The ratchet current rapidly becomes very small as $\tau \longrightarrow 0$, but when the particle does move from the neighborhood of the stable equilibrium point at 0 (same as $L$ ), it evidently finds it ever more pos- 
sible to go the larger distance $\alpha$ to the right under the influence of the larger net force than the shorter distance $L-\alpha$ pushed by the weaker net force.

Now consider the case $D=0$ and $V(x)$ piecewise linear as in Fig. 1(a). The previous asymptotic analysis is not applicable as indicated by the $\left|V^{\prime \prime}\right|$ terms in the prefactor in Eq. (22), undefined for the discontinuous force problem. In order to see the $D=0, \tau \rightarrow 0$ behavior when the ratchet force is piecewise constant we may directly evaluate the integrals in the exact solution to obtain a formula for $J$ as a function of the system parameters. This is a straightforward but exceptionally tedious exercise, the result of which is the exact result

$$
J=\frac{1}{Q}\left[1-\exp \left\{\frac{1}{\tau} \frac{E}{F^{2}-E^{2} / \alpha^{2}}-\frac{1}{\tau} \frac{E}{F^{2}-E^{2} /(L-\alpha)^{2}}\right\}\right],
$$

where

$$
\begin{aligned}
Q= & \frac{(L-\alpha)^{2}}{E}-\frac{\alpha^{2}}{E}+\frac{\tau L^{2} F^{2}}{E^{2}} \\
& \times\left(\exp \left\{-\frac{1}{\tau} \frac{E}{F^{2}-E^{2} /(L-\alpha)^{2}}\right\}-1\right) \\
& +\frac{\tau L^{2} F^{2}}{E^{2}} \exp \left\{\frac{1}{\tau} \frac{E}{F^{2}-E^{2} / \alpha^{2}}\right\} \\
& +\left(\frac{\alpha^{2}}{E}-\frac{(L-\alpha)^{2}}{E}-\frac{\tau L^{2} F^{2}}{E^{2}}\right) \\
& \times \exp \left\{\frac{1}{\tau} \frac{E}{F^{2}-E^{2} / \alpha^{2}}-\frac{1}{\tau} \frac{E}{F^{2}-E^{2} /(L-\alpha)^{2}}\right\},
\end{aligned}
$$

and $E$ is the height of the energy barrier as indicated in Fig. 1. The leading behavior in the $\tau \rightarrow 0$ limit is significantly simpler:

$$
J \sim \frac{E^{2}}{\tau L^{2} F^{2}} \exp \left\{-\frac{1}{\tau} \frac{E}{F^{2}-E^{2} / \alpha^{2}}\right\} .
$$

As for smooth potentials, this is also $O\left(e^{-C / \tau}\right)$ but with a qualitatively different prefactor which decreases as $F$ increases and increases as $E$ increases. The dominant term in the exponent is precisely $-\phi(\alpha)$, so this exponential aspect is apparently insensitive to the regularity of the ratchet potential and the relevant considerations above apply to the discontinuous force case as well.

Turning our attention to the $D \neq 0$ situation, consider the case of a smooth potential. The singular perturbation analysis of Eq. (18) proceeds with the ansatz that the probability densities may be expanded, for small $\tau$, in an asymptotic series

$$
\rho_{ \pm}(x) \sim \rho_{ \pm}^{(0)}(x)+\tau \rho_{ \pm}^{(1)}(x)+\tau^{2} \rho_{ \pm}^{(2)}(x)+\cdots,
$$

and similarly that $J$ has an asymptotic expansion

$$
J \sim J^{(0)}+\tau J^{(1)}+\tau^{2} J^{(2)}+\cdots .
$$

Inserting the expansion into the equations and examining them order-by-order, we can systematically compute the functions $\rho_{ \pm}^{(n)}(x)$. As usual in such singular perturbation problems, some of the equations at each order arise as inte- grability conditions for higher-order equations. This procedure is directly analogous to other singular perturbation analyses for Fokker-Planck equations, ${ }^{11}$ and the detailed calculation for another version of this kind of problem has been described in Ref. 5. The result of this lengthy calculation yields the first nonzero contribution to $J$ at third order in $\tau$.

$$
J \sim \frac{\tau^{3}}{Y Z}\left[\frac{F^{4}}{D^{3}} \int_{0}^{L} f(x)^{3} d x+\frac{F^{2}}{D} \int_{0}^{L} f(x) V^{\prime \prime}(x)^{2} d x\right],
$$

where

$$
Y=\int_{0}^{L} e^{V(x) / D} d x \quad \text { and } \quad Z=\int_{0}^{L} e^{-V(x) / D} d x
$$

This result indicates an exponentially large increase in the current, from $O\left(e^{-C / \tau}\right)$ to $O\left(\tau^{3}\right)$, for $D \neq 0$ as compared to the $D=0$ expression in Eq. (22). So, the presence of white noise greatly enhances the transport for fast external noise, even though the two fluctuation sources are (in this model) statistically independent. The extreme sensitivity to the white noise is indicated by the $Y$ factor in the denominator of the prefactor above: it diverges exponentially for small $D$ so the expression in Eq. (29) vanishes like $O\left(e^{-E / D}\right)$ as $D \rightarrow 0$. Of course the limits $D \rightarrow 0$ and $\tau \rightarrow 0$ do not commute in these formulas, but the physical effect is clear: a very small amount of white noise can result in orders of magnitude greater current when $\tau$ is small. What is perhaps more surprising, though, is the appearance of $V^{\prime \prime}(x)^{2}$ in Eq. (29). The second integral in Eq. (29) is undefined for discontinuous forces-even within the most liberal interpretations!-and it signals the appearance of different effects.

Consider now the $\tau \rightarrow 0$ problem for the piecewise linear potential with discontinuous force. The mathematical difference between the continuous and discontinuous force cases is the effect of the boundary conditions. As the asymptotic expansion in Eq. (27) is solved order by order, we impose periodic boundary conditions as $x$ varies between 0 and $L$. When the force is continuous, then the densities $\rho_{ \pm}^{(n)}(x)$ are continuous and this is a straightforward procedure. When the force is only piecewise continuous, say, on $(0, \alpha)$ and $(\alpha, L)$ as in Fig. 1(b), then the global periodic boundary conditions must be applied in pieces. The new feature that arises results from the fact that there are other asymptotic solutions to the perturbation equations which are not periodic on the full interval but which must now be included on the subintervals. The "new" solutions appear when we start with a more general ansatz for the asymptotic expansion of linearly independent solutions of the ordinary differential equations in Eq. (18), namely,

$$
\begin{aligned}
\rho_{ \pm}(x) \sim \exp & \left\{\frac{\psi(x)}{\sqrt{\tau}}\right\}\left[R_{ \pm}^{(0)}(x)+\sqrt{\tau} R_{ \pm}^{(1)}(x)\right. \\
& \left.+\tau R_{ \pm}^{(2)}(x)+\cdots\right],
\end{aligned}
$$

where the new function $\psi(x)$ is to be determined. This approach is inspired by the usual Wentzel-Kramers-Brillouin 
(WKB) approach to such problems, noting that Eq. (18) may be rewritten with the small parameter $\tau$ multiplying the highest-order derivative.

Inserting Eq. (31) into Eq. (18), we find the leading $O\left(\tau^{-1}\right)$ vector equation

$$
\left(\begin{array}{l}
0 \\
0
\end{array}\right)=\frac{1}{2 \tau}\left(\begin{array}{cc}
-1+2 D \psi^{\prime}(x)^{2} & 1 \\
1 & -1+2 D \psi^{\prime}(x)^{2}
\end{array}\right)\left(\begin{array}{l}
R_{+}^{(0)} \\
R_{-}^{(0)}
\end{array}\right) .
$$

The solvability condition determining $\psi(x)$ is the vanishing of the determinant of the matrix above:

$$
0=D \psi^{\prime}(x)^{2}\left(D \psi^{\prime}(x)^{2}-1\right) .
$$

Hence there are three possibilities for $\psi(x)$ :

$$
\psi(x)=\text { const } \quad \text { or } \quad \psi(x)= \pm \frac{x}{\sqrt{D}} .
$$

The constant $\psi$ solutions are those that arise in the smooth potential analysis (the constant may without loss of generality be taken to be zero). The new solutions have components dominated by boundary-layer-like functions

$$
\exp \left\{ \pm \frac{x}{\sqrt{D \tau}}\right\} \text {. }
$$

These functions are not periodic on the full interval and so do not play a role when the periodic boundary conditions may be imposed in one step, but there is no reason not to include them in the general solution on the subintervals. That is, the density takes the form of one linear combination,

$$
\begin{aligned}
\rho_{ \pm}(x) \sim & \sum_{k=0} \tau^{k / 2}\left[R_{ \pm}^{(k)}(x)+\exp \left\{\frac{+x}{\sqrt{D \tau}}\right\} N_{ \pm}^{(k)}(x)\right. \\
& \left.+\exp \left\{\frac{-x}{\sqrt{D \tau}}\right\} M_{ \pm}^{(k)}(x)\right],
\end{aligned}
$$

for $x \in(0, \alpha)$, and another,

$$
\begin{aligned}
\rho_{ \pm}(x) \sim \sum_{k=0} \tau^{k / 2}\left[r_{ \pm}^{(k)}(x)+\exp \left\{\frac{+x}{\sqrt{D \tau}}\right\} n_{ \pm}^{(k)}(x)\right. \\
\left.+\exp \left\{\frac{-x}{\sqrt{D \tau}}\right\} m_{ \pm}^{(k)}(x)\right],
\end{aligned}
$$

for $x \in(\alpha, L)$. As usual, the functions $R_{ \pm}^{(k)}, N_{ \pm}^{(k)}, M_{ \pm}^{(k)}$, $r_{ \pm}^{(k)}, n_{ \pm}^{(k)}$, and $m_{ \pm}^{(k)}(x)$ are determined by integrability conditions for higher-order terms in the expansion. The boundary conditions are then imposed, order by order, as continuity and jump conditions across $x=\alpha$ and $x=L$ (appropriately identified as $0^{-}$).

The mathematical complexity increases tremendously in comparison to the smooth potential case, and closed analytic solutions are all but impossible to obtain. However, progress can be made with the help of computer algebra routines guided by direct numerical solutions of the differential equations, and to date we have algebraically computed terms contributing to the current up to and including $O\left(\tau^{3 / 2}\right)$. To that

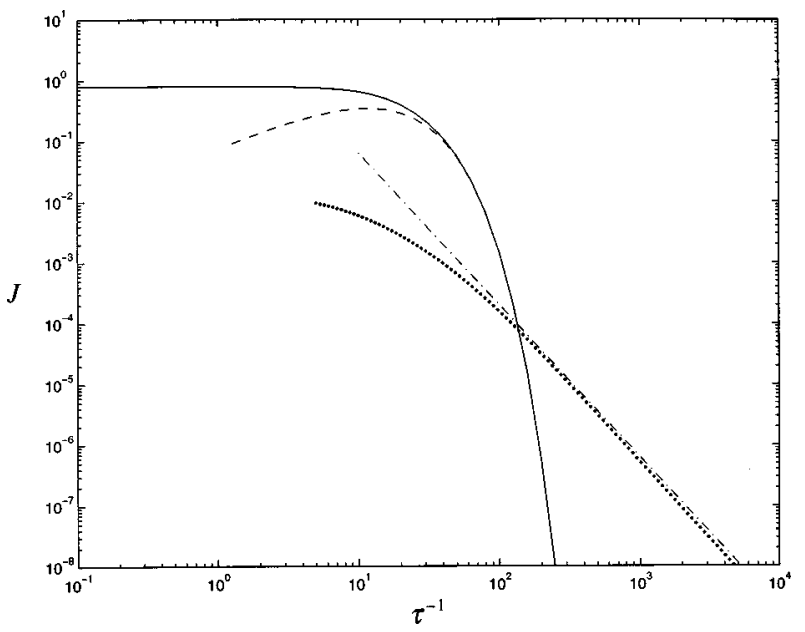

FIG. 3. Current versus fluctuation rate $1 / \tau$ on a $\log \log$ scale. The $\tau^{5 / 2}$ asymptotic scaling as $\tau \rightarrow 0$ is visible in the data from direct numerical solution of Eq. (18) for the piecewise linear potential as in Fig. 1(b) with parameters $D=1, E=1, F=5, \alpha=3 / 4$, and $L=1$ (discrete points). The dash-dot line $\sim \tau^{5 / 2}$ is drawn to guide the eye. The solid line is the $D=0$ current versus fluctuation rate for the piecewise linear potential from the exact result in Eqs. (24) and (25) with the other parameters the same, i.e., $E=1, \quad F=5, \alpha=3 / 4$, and $L=1$. The leading zero-diffusion-fast-noise asymptotic term in Eq. (26) is also plotted (dashed line).

order there is no current. Direct numerical solution of the differential equations, as shown in Fig. 3, indicates that as $\tau \rightarrow 0$,

$$
J \sim \tau^{5 / 2} .
$$

We must await the outcome of the computer-aided algebra analysis to identify the prefactor. This "anomalous" scaling is to be contrasted with $\tau^{3}$ scaling (see Ref. 5) for smooth ratchet potentials.

\section{SUMMARY AND DISCUSSION}

We have performed a detailed investigation of the fastnoise limit of the nonequilibrium fluctuation-induced current in the simplest model of a stochastic ratchet. In particular we studied the dichotomously forced overdamped average drift of a particle diffusing in an anisotropic periodic potential, focusing on the asymptotic behavior of the probability current as the time scale $\tau$ of the applied force approached zero. The analysis uncovered some interesting and relatively subtle aspects of the mathematics and the physics.

In the absence of white noise perturbations, we found that the $O\left(e^{-C / \tau}\right)$ residual current becomes independent of the "uphill" portion of the ratchet potential. This suggests that the transitions, rare though they may be, become more one sided in the $\tau \rightarrow 0$ limit. The analysis was sensitive to details of the regularity of the potential. By comparison of the full explicit exact solution of the piecewise linear ratchet potential problem with an asymptotic analysis of the exact solution for smooth potentials, we observed that the $O\left(e^{-C / \tau}\right)$ factor is robust while the prefactor adopts very different forms in the two cases. This result is not altogether surprising in view of the similar dependence of the prefactor on the smoothness of the potential in the Kramers rate problem. There, the different low-temperature (small $D$ ) prefac- 
tors arise in a well-understood way from different applications of Langrange's method to the integral expression for the mean exit time. In the case studied here, the small noise correlation time $\tau$ played the mathematical role of a temperature or diffusion coefficient in this regard. Beyond such strictly theoretical considerations, these exact mathematical results for the $D=0$ case may also be useful as benchmark calculations for checking numerical solutions and simulations - and possibly someday for calibrating experimental devices.

White noise had a profound effect on the magnitude of the current in the small $\tau$ limit: the current was elevated from $O\left(e^{-C / \tau}\right)$ to $O\left(\tau^{3}\right)$ in the presence of additional white noise fluctuations; see Fig. 3. Apparently the two noises acting together can potentially generate far more organized motion than either can alone, even though they are statistically independent noise sources in this model. This constructive cooperation between the random fluctuations is reminiscent of fundamental phenomena in the more widely studied area of stochastic resonance. ${ }^{12}$ In each situation the combined effect of ostensibly independent perturbations results in enhanced organized system response. The example studied here provides a quantitative illustration of this effect in a simple physical model.

Beyond the ratchet current induced by the two noises, our analysis has uncovered a new quantitative aspect of the interaction of the noises: they combine to produce a new (small) length scale,

$$
\varepsilon=\sqrt{D \tau}
$$

This small scale emerges as the boundary layer thickness for the probability densities in the neighborhood of discontinuities in the force, leading to typical $O\left(e^{-|x| / \varepsilon}\right)$ boundary layer correction terms. This is not an unnatural length scale; it is the typical distance the particle may diffuse between switches of the dichotomous force. It is of special interest for the purposes of this discussion that $\varepsilon$ depends fundamentally on the two different noise sources; there are no such bound- ary layers in the absence of one source of fluctuations or the other. The qualitative effect of these terms on the nonequilibrium current is profound. The scaling exponent for the current in the small $\tau$ limit changes from 3 for smooth potentials to $\frac{5}{2}$ when the boundary layers appear. Such dramatic mathematical effects of the potential regularity are surprising when one notes that there is $O(1)$ diffusion in the equations. For simple diffusion problems most quantities of physical interest are quantitatively robust to such considerations. It remains to be seen if such quantitative aspects of the cooperative effects of independent noises plays a role in other problems in physics or engineering.

\section{ACKNOWLEDGMENTS}

This research was supported by NSF Grant No. PHY 96-96187 with a REU Supplement, and NSF Grant No. DMS 97-09494.

${ }^{1}$ H. A. Kramers, Physica (Utrecht) 7, 284 (1940).

${ }^{2}$ H. Risken, The Fokker Planck Equations (Springer, Berlin, 1984).

${ }^{3}$ M. Magnasco, Phys. Rev. Lett. 71, 1477 (1993).

${ }^{4}$ (a) C. R. Doering, W. Horsthemke, and J. Riordan, Phys. Rev. Lett. 72, 2984 (1994); (b) R. Bartussek, P. Hänggi, and J. G. Kissner, Europhys. Lett. 28, 459 (1994); (c) M. Bier, Phys. Lett. A 211, 12 (1996).

${ }^{5}$ T. C. Elston and C. R. Doering, J. Stat. Phys. 83, 359 (1996).

${ }^{6}$ R. D. Astumian and M. Bier, Phys. Rev. Lett. 72, 1766 (1994); J. Prost, J. Chauwin, L. Peliti, and A. Ajdari, ibid. 72, 2652 (1994).

${ }^{7}$ C. Peskin and G. Oster, Biophys. J. 68, 202s (1995).

${ }^{8}$ J. Rousselet, L. Salome, A. Ajdari, and J. Prost, Nature (London) 370 (1994); L. P. Faucheux, L. S. Bordieu, P. D. Kaplan, and A. J. Libchaber, Phys. Rev. Lett. 74, 1504 (1995).

9 (a) C. R. Doering, Nuovo Cimento D 17, 685 (1995); (b) P. Hänggi and R. Bartussel, Lect. Notes Phys. 476, 294 (1996); (c) F. Jülicher, A. Ajdari, and J. Prost, Rev. Mod. Phys. 69, 1269 (1997); (d) R. D. Astumian, Science 276, 917 (1997).

${ }^{10}$ J. D. Murray, Asymptotic Analysis (Springer, New York, 1984).

${ }^{11} \mathrm{~W}$. Horsthemke and R. Lefever, Noise Induced Transitions (Springer, New York, 1984); singular perturbation theory for more general discrete Markovian noise is developed in M. M. Klosek, B. J. Matkowsy, and Z. Schuss, SIAM (Soc. Ind. Appl. Math.) J. Appl. Math. 49, 1811 (1989).

${ }^{12} \mathrm{See}$, for example, the other relevant contributions to this issue of Chaos or the recent review: L. Gammaitoni, P. Hänggi, P. Jung, and F. Marchesoni, Rev. Mod. Phys. 70, 223 (1998). 